\title{
Photoacoustic endoscopic imaging of the rabbit mediastinum
}

Joon-Mo Yang, Christopher Favazza, Ruimin Chen, Junjie Yao, Xin Cai, et al.

Joon-Mo Yang, Christopher Favazza, Ruimin Chen, Junjie Yao, Xin Cai, Chiye Li, Konstantin Maslov, Qifa Zhou, K. Kirk Shung, Lihong V. Wang, "Photoacoustic endoscopic imaging of the rabbit mediastinum," Proc. SPIE 8581, Photons Plus Ultrasound: Imaging and Sensing 2013, 85813V (4 March 2013); doi: $10.1117 / 12.2004980$

SPIE. Event: SPIE BiOS, 2013, San Francisco, California, United States 


\title{
Photoacoustic endoscopic imaging of the rabbit mediastinum
}

\author{
Joon-Mo Yang ${ }^{1}$, Christopher Favazza ${ }^{1}$, Ruimin $\mathrm{Chen}^{2}$, Junjie $\mathrm{Yao}^{1}$, Xin $\mathrm{Cai}^{1}$, Chiye $\mathrm{Li}^{1}$, $\mathrm{Konstantin}$ \\ Maslov $^{1}$, Qifa Zhou ${ }^{2}$, K. Kirk Shung ${ }^{2}$, and Lihong V. Wang ${ }^{1 *}$ \\ ${ }^{1}$ Optical Imaging Laboratory, Department of Biomedical Engineering, Washington University in St. \\ Louis, One Brookings Drive, Campus Box 1097, St. Louis, Missouri, 63130, USA \\ ${ }^{2}$ Ultrasonic Transducer Resource Center, Department of Biomedical Engineering, University of \\ Southern California, 1042 Downey Way, University Park, DRB 130, Los Angeles, CA 90089, USA
}

\begin{abstract}
Like ultrasound endoscopy, photoacoustic endoscopy (PAE) could become a valuable addition to clinical practice due to its deep imaging capability. Results from our recent in vivo transesophageal endoscopic imaging study on rabbits demonstrate the technique's capability to image major organs in the mediastinal region, such as the lung, trachea, and cardiovascular systems. Here, we present various features from photoacoustic images from the mediastinal region of several rabbits and discuss possible clinical contributions of this technique and directions of future technology development.
\end{abstract}

Keywords: Photoacoustic endoscopy, endoscopic ultrasound, upper gastrointestinal tract, transesophageal imaging, rabbit mediastinum.

\section{INTRODUCTION}

Photoacoustic endoscopy (PAE) ${ }^{1-7}$ is a promising tomographic endoscopy modality that provides a unique combination of functional optical contrast and high spatial resolution at clinically relevant depths, far exceeding the penetration depths of conventional high-resolution optical imaging modalities ${ }^{8-17}$. Moreover, it can provide unprecedented physiological and functional information of the target tissue with the aid of endogenous or exogenous contrast agents ${ }^{18-}$ 27. With these attributes, PAE's potential clinical contributions could rival or exceed those of current ultrasound endoscopy, also called endoscopic ultrasound (EUS) ${ }^{28,29}$.

Results from our recent transesophageal imaging study on rabbits demonstrate the technique's ability to image major organs in the mediastinal region, such as the lung, trachea, and cardiovascular systems (Nature Medicine, 2012) ${ }^{3}$. Also, the simultaneous, multi-wavelength spectral imaging capability of our system enables the provision of a wealth of functional information, such as the total hemoglobin concentration, the oxygen saturation of hemoglobin, and the dynamics of the lymphatic system. In this paper, we present features from photoacoustic (PA) and ultrasonic (US) images from the mediastinal regions of two rabbits acquired in vivo and discuss the possible clinical contributions of this technique and directions of future technology development.

\section{MATERIALS AND METHODS}

\subsection{8-mm diameter integrated photoacoustic and ultrasonic endoscopic probe and system}

For this study, we utilized the 3.8-mm diameter probe based endoscopic system reported in Nature Medicine ${ }^{3}$. Figure 1 shows the endoscope and its peripheral systems, composed of a micromotor driver circuit, a delay generator, a laser system, a US pulser-receiver including an amplifier, a data acquisition (DAQ) card, and a computer for recording signals and displaying images.

*Corresponding author: lhwang@biomed.wustl.edu

Photons Plus Ultrasound: Imaging and Sensing 2013, edited by Alexander A. Oraevsky, Lihong V. Wang, Proc. of SPIE Vol. 8581, 85813V · (C) 2013 SPIE · CCC code: 1605-7422/13/\$18 - doi: 10.1117/12.2004980 


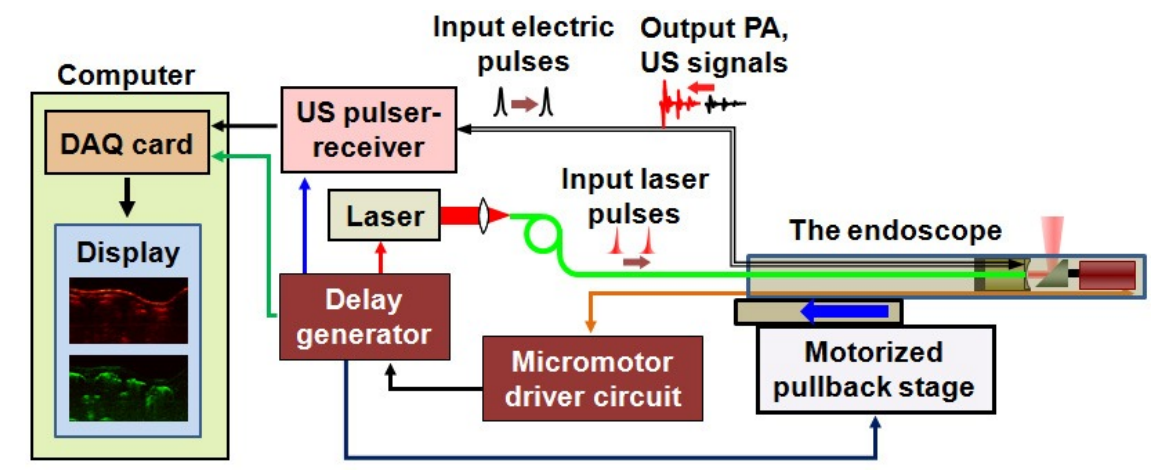

Figure 1. Block diagram showing the 3.8-mm diameter endoscopic probe and its connection to peripheral systems.

For PA imaging, laser pulses ( $584 \mathrm{~nm}, \sim 10 \mathrm{~ns}$ pulse width, $\sim 0.3 \mathrm{~mJ} /$ pulse) from a tunable dye laser (Cobra HRR, Sirah), pumped by a solid-state, diode-pumped Nd:YLF laser (INNOSLAB IS811-E, EdgeWave), are guided by a multimode optical fiber (BFL22-365, Thorlabs) and emitted through the central hole of a single element focused US transducer $\left(\mathrm{LiNbO}_{3}, \sim 36 \mathrm{MHz}, 65 \%\right.$ fractional bandwidth), which is coaxially aligned with the optical fiber. After exiting the fiber, the laser beams are further directed to the target tissue by a scanning mirror, and finally generate PA waves once absorbed by the target tissue. The PA waves that propagate to the scanning mirror are reflected by the same mirror, sent to the US transducer, converted into electrical signals, amplified by the US pulser-receiver (5072PR, Panametrics), and digitally recorded by the DAQ card (NI PCI-5124, National Instruments). For US imaging, we utilized the same US pulser-receiver which provided sharp electric pulses to the US transducer to generate acoustic pulses for US imaging and also amplified the US and PA signals detected by the transducer. With the endoscopic system, we acquired volumetric PA and US images with a B-scan frame rate of $\sim 4 \mathrm{~Hz}$. More information on the endoscope's structure is available in our previous reports ${ }^{3}$.

With the laser fluences used in these experiments, the endoscope's maximal radial imaging depth was $\sim 7 \mathrm{~mm}$ from the endoscope's surface, and the angular field-of-view was limited to approximately $270^{\circ}$, due to partial blocking by the probe housing. Experimentally measured highest PA and US resolutions in the focal zone of the transducer were respectively $\sim 55 \mu \mathrm{m}$ and $\sim 30 \mu \mathrm{m}$ in the axial direction, and $\sim 80 \mu \mathrm{m}$ and $\sim 60 \mu \mathrm{m}$ in the transverse direction, but the transverse resolution varied with target distance.

\subsection{Animal experiment}

We transesophageally imaged the mediastinum of two adult New Zealand white rabbits ( $\sim 4 \mathrm{~kg}$, Myrtles Rabbitry). The animals were fasted, beginning $\sim 12 \mathrm{hr}$ before the experiments, to reduce the likelihood of ingesta in the imaged gastrointestinal tracts. Before starting the endoscopic imaging experiments, we first anesthetized the rabbits with 35-50 $\mathrm{mg} / \mathrm{kg}$ of ketamine and 5-10 $\mathrm{mg} / \mathrm{kg}$ of xylazine (IM). While anesthetized, the rabbit was intubated and supplied with maintenance gas for anesthesia (1.5-3.0\% isoflurane). An endotracheal tube cuff was inserted into the trachea and inflated to prevent aspiration of water into the lung. The rabbit was placed on an inclined stage $\left(\sim 10^{\circ}\right)$ in supine position. Just prior to probe insertion, water was introduced into the esophagus and stomach, using an enteral feeding syringe connected to a rubber feeding tube $(8-12 \mathrm{~F})$. The water provided the necessary acoustic coupling and functioned as a lubricant during the imaging procedure. After the stomach and esophagus were filled with water, the endoscopic probe was inserted through the mouth and advanced $\sim 30 \mathrm{~cm}$ into the esophagus. Simultaneous PA and US imaging were immediately initiated. While acquiring images, the probe was slowly and mechanically pulled out of the esophagus using a motorized translation stage and with a constant pullback speed of $\sim 160 \mu \mathrm{m} / \mathrm{s}$. Each imaging session required a scanning time of $\sim 10 \mathrm{~min}$. About 2,800 B-scan slices at $\sim 40 \mu \mathrm{m}$ intervals were recorded for each volumetric set of PA imaging at $584 \mathrm{~nm}$ and US imaging. Throughout the experiment, the rabbit's anesthesia level and vital signs were continuously monitored. After the experiment, the rabbit was euthanized by an overdose of sodium pentobarbital (150 $\mathrm{mg} / \mathrm{kg}$ ) injected in the marginal ear vein. 
All procedures in the animal experiments followed the protocol approved by the Institutional Animal Care and Use Committee at Washington University in St. Louis.

\section{RESULTS}

In Figure 2, we present two sets of coregistered radial-maximum amplitude projection (RMAP) images showing the PA $(\mathbf{a}-\mathbf{b})$ and US (c-d) image features of the mediastinal regions of the two rabbits acquired in vivo. Each image was processed from a C-scan data set covering a cylindrical volume $\sim 11 \mathrm{~cm}$ long with a $\sim 18 \mathrm{~mm}$ diameter, excluding signals generated from the esophagi (wall thickness $\sim 1 \mathrm{~mm}$ ) to show anatomical structures in the mediastinal regions only. The PA structural images $(\mathbf{a}-\mathbf{b})$ were created from the PA data acquired at a $584 \mathrm{~nm}$ laser wavelength, in which the PA signal is only sensitive to the total hemoglobin concentration. The US images (c-d) represent the echogenicity distribution of the imaged structures like the conventional pulse-echo imaging that detects acoustic waves reflected from target tissue.
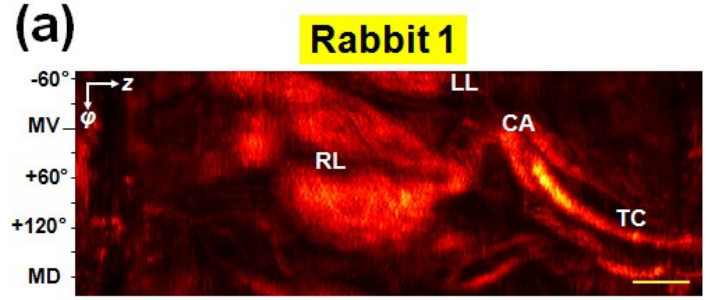

(c)

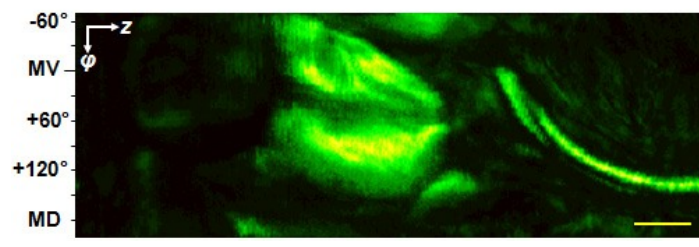

(b)

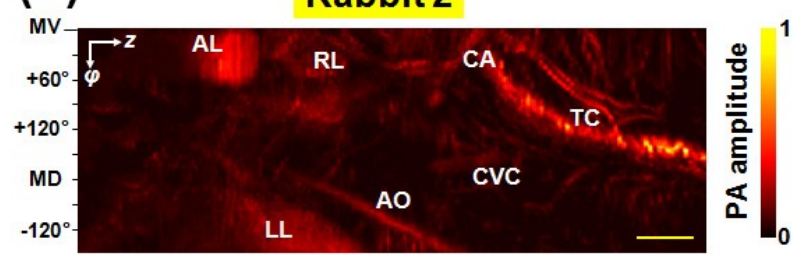

(d)

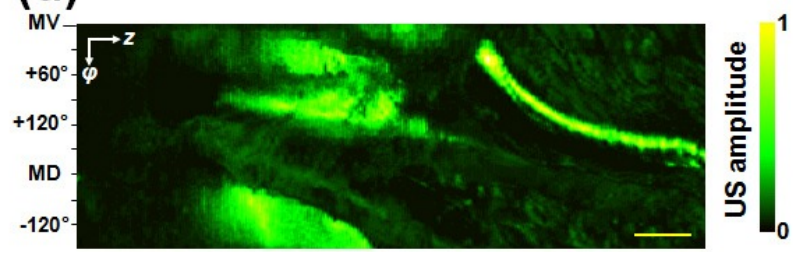

Figure 2. RMAP images of the mediastinal regions of the two rabbits (views from the inside of the esophagus). The leftand right-hand sides of these RMAP images correspond to the lower and upper esophagus, respectively. (a,b) Normalized PA-RMAP image showing the total hemoglobin distribution, with the esophageal signals excluded during the RMAP construction. AL, accessory lobe; LL, left lobe; RL, right lobe of the lung; AO, aorta; CVC, caudal vena cava; CA, carina; TC, trachea. (c,d) Coregistered US-RMAP images showing the echogenicity distribution for the organs presented in a and b, respectively. In each image, the vertical $\varphi$-axis corresponds to the angular range of $270^{\circ}$, and the horizontal $z$-axis corresponds to the pullback length of $11 \mathrm{~cm}$. The approximate mid-ventral (MV) position and angular measures from the MV are marked along the vertical $\varphi$-axis, where the positive and negative values correspond to the right and left sides of the animal, and MD denotes the mid-dorsal position. The scale bars represent $1 \mathrm{~cm}$ for the horizontal direction only.

The two PA images (a-b) commonly show the structures of major organs, such as the left lobe (LL), the right lobe (RL) of the lung, the carina (CA), and the trachea (TC), in the mediastinal regions. However, the PA image acquired from Rabbit 2 shows the accessory lobe (AL), the aorta (AO), and caudal vena cava (CVC) clearly, which were also observed in the previous PA image reported in our recent paper (Nature Medicine, 2012) ${ }^{3}$. These major structures were surgically validated after the endoscopic imaging procedures.

To understand the three-dimensional configuration of the imaged structures in Figure 2, we produced two volumetric images and present them in Figure 3. In these images, the contrast differences between PA and US imaging are clearly shown. 


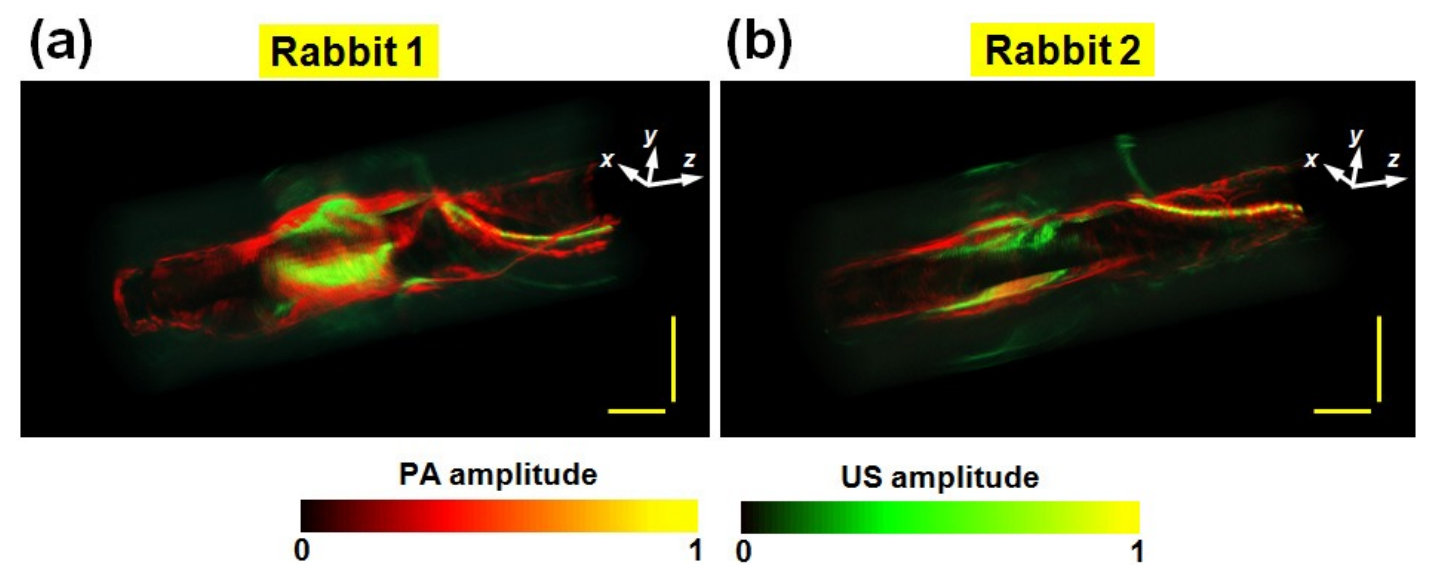

Figure 3. Three-dimensionally-rendered coregistered PA-US mediastinum images of the two rabbits, processed from the data presented in Figure 2. Each image covers a $\sim 11 \mathrm{~cm}$ range with a $\sim 18 \mathrm{~mm}$ diameter. The red color corresponds to the PA signals and the green color to the US signals. The left-hand side $(-z)$ of each image corresponds to the lower esophagus of the animal. The horizontal and vertical scale bars represent $1 \mathrm{~cm}$ and $5 \mathrm{~mm}$, respectively.

\section{DISCUSSION}

The presented results show the integrated endoscopic system's complementary contrast production ability and the importance of the dual-mode imaging for the better understanding of morphologic structures of the target tissue. Although traditional EUS imaging technique is used for imaging the human mediastinum, as we presented in this paper, PAE could provide unprecedented image information in clinical applications, with superior image contrast. Here, we summarize several unique features of PAE over the traditional EUS imaging technique, including the provisions of (1) the optical-absorption-based contrast over a wide spectral range with high spatial resolution at super depths, exceeding the limit of conventional optical microscopy; (2) high resolution (vascular) imaging with intrinsic contrast; (3) functional imaging through spectral unmixing, such as total hemoglobin concentration and oxygen concentration; (4) greater flexibility in employing existing and novel contrast agents compared to EUS, with the potential for molecular imaging; (5) high contrast lymphatic system imaging with the aid of exogenous contrast agents; (6) simultaneous dualmodality imaging with complementary contrast (PAE-EUS).

So far, we have focused on developing endoscopic probes that can be used for imaging the gastrointestinal tract, for which several-millimeter diameter probe sizes are acceptable. However, to apply this technique for intravascular imaging or use in the instrument channel of a video endoscope, related catheters should be further miniaturized, typically $\sim 1 \mathrm{~mm}$ for intravascular imaging and $\sim 2.5 \mathrm{~mm}$ to use in the instrument channel. For clinical applications, achieving a real-time image frame rate (typically $\sim 30 \mathrm{~Hz}$ ) is essential to acquire volumetric images minimizing motion artifacts. Also, implementing a flexible-shaft based endoscope would promote broadening the applications of this technique. Development of array transducer-based electronic scanning endoscopic systems would be a promising direction because such systems could provide cross-sectional images with a single shot of a laser pulse and also would enable achieving a high image frame rate. In our recent endoscopic studies ${ }^{3,4}$, we demonstrated acoustic resolution PA endoscopes with image resolution determined by the acoustic parameters. However, the lateral resolution could be greatly improved if an optical focusing method were applied, as commonly used in optical-resolution photoacoustic microscopy ${ }^{21,30-34}$.

\section{ACKNOWLEDGEMENT}

We thank Seema Dahlheimer for her attentive reading of the manuscript. This work was sponsored in part by National Institutes of Health grants R01 CA157277, R01 NS46214 (BRP), R01 EB000712, R01 EB008085, and U54 CA136398 (Network for Translational Research). L.W. has a financial interest in Microphotoacoustics, Inc. and Endra, Inc., which, however, did not support this work. 


\section{REFERENCES}

1. Yang, J.M., et al. Photoacoustic endoscopy. Opt Lett 34, 1591-1593 (2009).

2. Yang, J.M., et al. Volumetric photoacoustic endoscopy of upper gastrointestinal tract: ultrasonic transducer technology development Proc. SPIE 7899, 78990D (2011).

3. Yang, J.M., et al. Simultaneous functional photoacoustic and ultrasonic endoscopy of internal organs in vivo. Nat Med 18, 1297$1302(2012)$

4. Yang, J.M., et al. A 2.5-mm diameter probe for photoacoustic and ultrasonic endoscopy. Opt Express 20, 23944-23953 (2012).

5. Karpiouk, A.B., Wang, B. \& Emelianov, S.Y. Development of a catheter for combined intravascular ultrasound and photoacoustic imaging. Rev Sci Instrum 81, 014901 (2010).

6. Jansen, K., van der Steen, A.F., van Beusekom, H.M., Oosterhuis, J.W. \& van Soest, G. Intravascular photoacoustic imaging of human coronary atherosclerosis. Opt Lett 36, 597-599 (2011).

7. Wang, B., et al. Intravascular photoacoustic imaging of lipid in atherosclerotic plaques in the presence of luminal blood. Opt Lett 37, 1244-1246 (2012).

8. Tearney, G.J., et al. In vivo endoscopic optical biopsy with optical coherence tomography. Science 276, 2037-2039 (1997).

9. Kiesslich, R., et al. Confocal laser endoscopy for diagnosing intraepithelial neoplasias and colorectal cancer in vivo. Gastroenterology 127, 706-713 (2004).

10. Yun, S.H., et al. Comprehensive volumetric optical microscopy in vivo. Nat Med 12, 1429-1433 (2006).

11. Adler, D.C., et al. Three-dimensional endomicroscopy using optical coherence tomography. Nat Photon 1, 709-716 (2007).

12. Kim, P., et al. In vivo wide-area cellular imaging by side-view endomicroscopy. Nat Methods 7, 303-305 (2010).

13. Li, X., Chudoba, C., Ko, T., Pitris, C. \& Fujimoto, J.G. Imaging needle for optical coherence tomography. Opt Lett 25, 1520$1522(2000)$.

14. Liu, X., Cobb, M.J., Chen, Y., Kimmey, M.B. \& Li, X. Rapid-scanning forward-imaging miniature endoscope for real-time optical coherence tomography. Opt Lett 29, 1763-1765 (2004).

15. Yoo, H., et al. Intra-arterial catheter for simultaneous microstructural and molecular imaging in vivo. Nat Med 17, $1680-1684$ (2011).

16. Winkler, A.M., et al. In vivo, dual-modality OCT/LIF imaging using a novel VEGF receptor-targeted NIR fluorescent probe in the AOM-treated mouse model. Mol Imaging Biol 13, 1173-1182 (2011).

17. Qiu, L., et al. Multispectral scanning during endoscopy guides biopsy of dysplasia in Barrett's esophagus. Nat Med 16, 603-606 (2010).

18. Oraevsky, A.A. \& Karabutov, A.A. Optoacoustic Tomography. in Biomedical Photonics Handbook, Vol. PM125 (ed. Vo-Dinh, T.) 3401-3434 (CRC Press, New York, 2003).

19. Wang, L.V. Multiscale photoacoustic microscopy and computed tomography. Nat Photonics 3, 503-509 (2009).

20. Wang, L.V. \& Hu, S. Photoacoustic tomography: in vivo imaging from organelles to organs. Science 335, 1458-1462 (2012).

21. Yao, J., Maslov, K.I., Zhang, Y., Xia, Y. \& Wang, L.V. Label-free oxygen-metabolic photoacoustic microscopy in vivo. $J$ Biomed Opt 16, 076003 (2011).

22. Beard, P. Biomedical photoacoustic imaging. Interface Focus 1, 602-631 (2011).

23. Favazza, C.P., Cornelius, L.A. \& Wang, L.V. In vivo functional photoacoustic microscopy of cutaneous microvasculature in human skin. J Biomed Opt 16, 026004 (2011).

24. Yao, J., Maslov, K.I., Shi, Y., Taber, L.A. \& Wang, L.V. In vivo photoacoustic imaging of transverse blood flow by using Doppler broadening of bandwidth. Opt Lett 35, 1419-1421 (2010).

25. Danielli, A., Favazza, C.P., Maslov, K. \& Wang, L.V. Single-wavelength functional photoacoustic microscopy in biological tissue. Opt Lett 36, 769-771 (2011).

26. Wang, Y. \& Wang, L.V. Forster resonance energy transfer photoacoustic microscopy. J Biomed Opt 17, 086007 (2012).

27. Gao, L., et al. Single-cell photoacoustic thermometry. J Biomed Opt 18, 26003 (2013).

28. Dietrich, C. (ed.) Endoscopic Ultrasound: An Introductory Manual and Atlas, (Thieme, New York, 2006).

29. Kaul, V., et al. Interventional EUS. Gastrointest Endosc 72, 1-4 (2010).

30. Maslov, K., Zhang, H.F., Hu, S. \& Wang, L.V. Optical-resolution photoacoustic microscopy for in vivo imaging of single capillaries. Opt Lett 33, 929-931 (2008).

31. Song, L., Maslov, K. \& Wang, L.V. Section-illumination photoacoustic microscopy for dynamic 3D imaging of microcirculation in vivo. Opt Lett 35, 1482-1484 (2010).

32. Hu, S., Maslov, K. \& Wang, L.V. Second-generation optical-resolution photoacoustic microscopy with improved sensitivity and speed. Opt Lett 36, 1134-1136 (2011).

33. Wang, L., Maslov, K., Yao, J., Rao, B. \& Wang, L.V. Fast voice-coil scanning optical-resolution photoacoustic microscopy. Opt Lett 36, 139-141 (2011).

34. Yao, J., et al. Wide-field fast-scanning photoacoustic microscopy based on a water-immersible MEMS scanning mirror. J Biomed Opt 17, 080505-080501 (2012). 